\title{
Strongly correlating liquids and their isomorphs
}

\author{
Ulf R. Pedersen ${ }^{\text {a }}$, Nicoletta Gnan ${ }^{\text {b }}$, Nicholas P. Bailey ${ }^{\text {b }}$, Thomas B. Schrøder ${ }^{\text {b }}$, Jeppe C. Dyre ${ }^{\text {b,* }}$ \\ a Department of Chemistry, University of California, Berkeley, CA 94720, USA \\ b DNRF Center "Glass and Time", IMFUFA, Dept. of Sciences, Roskilde University, P.O. Box 260, DK-4000 Roskilde, Denmark
}

\section{A R T I C L E I N F O}

Available online 16 September 2010

\section{Keywords:}

Glass transitions;

Viscous liquids;

Strongly correlating liquids;

Isomorphs

\begin{abstract}
A B S T R A C T
This paper summarizes the properties of strongly correlating liquids, i.e., liquids for which the virial/potential energy correlation coefficient is above 0.9 for equilibrium fluctuations in the NVT ensemble. The definition and properties of strongly correlating liquids' isomorphs are given, and various isomorph invariants are discussed. The cause of strong virial/potential energy correlations is also discussed, and it is argued that strongly correlating liquids are not merely to be thought of as approximate inverse power-law liquids. The experimental predictions for strongly correlating glass-forming liquids include: i) density scaling; ii) isochronal superposition; iii) that there is a single function of frequency from which all frequencydependent viscoelastic response functions may be calculated; iv) that strongly correlating liquids are approximately single-parameter liquids with close to unity Prigogine-Defay ratio; v) that the fictive temperature initially decreases for an isobaric temperature up jump. The paper also briefly discusses the "isomorph filter", which provides a necessary condition for universality of theories for the non-Arrhenius temperature dependence of the relaxation time.
\end{abstract}

(c) 2010 Elsevier B.V. All rights reserved.

\section{Introduction}

After initial brief reports in early 2008 of the existence of the class of strongly correlating liquids $[1,2]$ these liquids were described in four comprehensive publications that appeared later in 2008 and in 2009 in the Journal of Chemical Physics [3-6]. The present paper briefly summarizes the properties and characteristics of strongly correlating liquids as detailed in Refs. [3-6] and presents several new computer simulations. We list a number of experimental predictions for strongly correlating liquids, focusing on glass-forming liquids. The main message is that the class of strongly correlating liquids, which includes the van der Waals and metallic liquids, are simpler than liquids in general. This is consistent with the long known observation that hydrogen-bonded liquids have several peculiar properties.

\section{Strong virial/potential energy correlations in liquids}

Consider a system of $N$ particles in volume $V$ at temperature $T$. The virial $W$ is defined by writing the pressure $p$ is a sum of the ideal gas term $N k_{B} T / V$ and a term reflecting the interactions, that is

$p V=N k_{B} T+W$.

\footnotetext{
* Corresponding author.

E-mail address: dyre@ruc.dk (J.C. Dyre).
}

If $U\left(\mathbf{r}_{1}, \ldots, \mathbf{r}_{N}\right)$ is the potential energy function, the virial (which has dimension of energy) is defined [7-11] by

$W\left(\mathbf{r}_{1}, \ldots, \mathbf{r}_{N}\right)=-1 / 3 \sum_{i} \mathbf{r}_{i} \cdot \nabla_{\mathbf{r}_{i}} U\left(\mathbf{r}_{1}, \ldots, \mathbf{r}_{N}\right)$.

Eq. (1) describes thermodynamic averages, but it also applies for the instantaneous values if the virial is defined by Eq. (2) and the temperature is defined from the kinetic energy in the usual fashion [7-11].

Consider now thermal equilibrium fluctuations at a given state point studied at fixed volume. If $\Delta U$ is the instantaneous potential energy minus its average and $\Delta W$ the same for the virial at any given state point, the $W U$ correlation coefficient $R$ is defined by (where sharp brackets denote equilibrium NVT ensemble averages)

$R=\frac{<\Delta W \Delta U>}{\sqrt{<(\Delta W)^{2}><(\Delta U)^{2}>}}$.

By the Cauchy-Schwarz inequality the correlation coefficient obeys $-1 \leq R \leq 1$. We define strongly correlating liquids by the condition [3]

$R \geq 0.9$.

The correlation coefficient is state-point dependent, but for all of the several liquids we have so far studied by simulation $[3,5,12] R$ is either above 0.9 in a large part of the phase diagram, or not at all. 
Fig. 1 shows two examples of constant-volume thermal equilibrium fluctuations of virial and potential energy for two model systems, the standard Lennard-Jones ( $\mathrm{LJ}$ ) liquid and the Wahnström binary Lennard-Jones mixture [13]. In both cases there are strong virial/ potential energy correlations. In (b) one observes two striking dips in the potential energy; these dips reflect the existence of transient clusters characterized by the same short-range order as the crystal [14]. During the dips virial and potential energy also correlate strongly. Actually, the $W U$ correlation even survives crystallization [4]. The property of strong virial/potential energy correlation is quite robust even complex systems like biological membranes may exhibit strong correlations [15].

One way to illuminate the correlations is to plot instantaneous values of virial and potential energy versus one another in so-called scatter plots. Fig. 2(a) shows an example of this with data taken from a simulation of the Kob-Andersen binary Lennard-Jones (KABLJ) liquid [16]. This has become the standard liquid for studying viscous liquid dynamics, because it is difficult to crystallize (which requires simulating for more than $100 \mu$ s (Argon units)[17]). The "slope" $\gamma$ of the scatter plot gives the proportionality constant of the fluctuations according to

$\Delta W(t) \cong \gamma \Delta U(t)$.

(a)

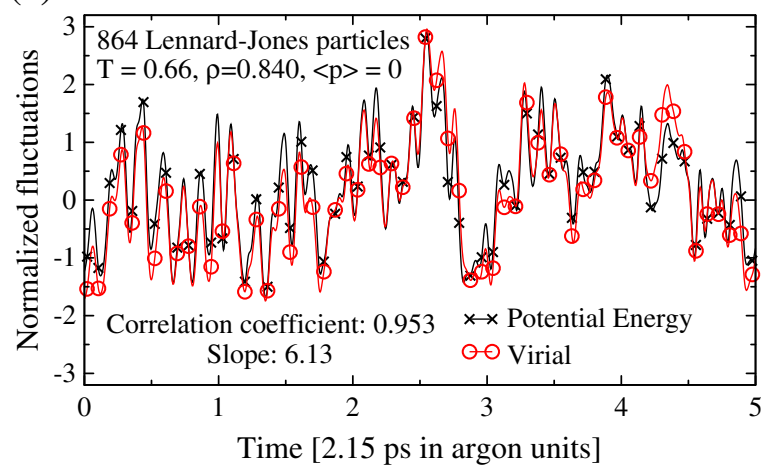

(b)

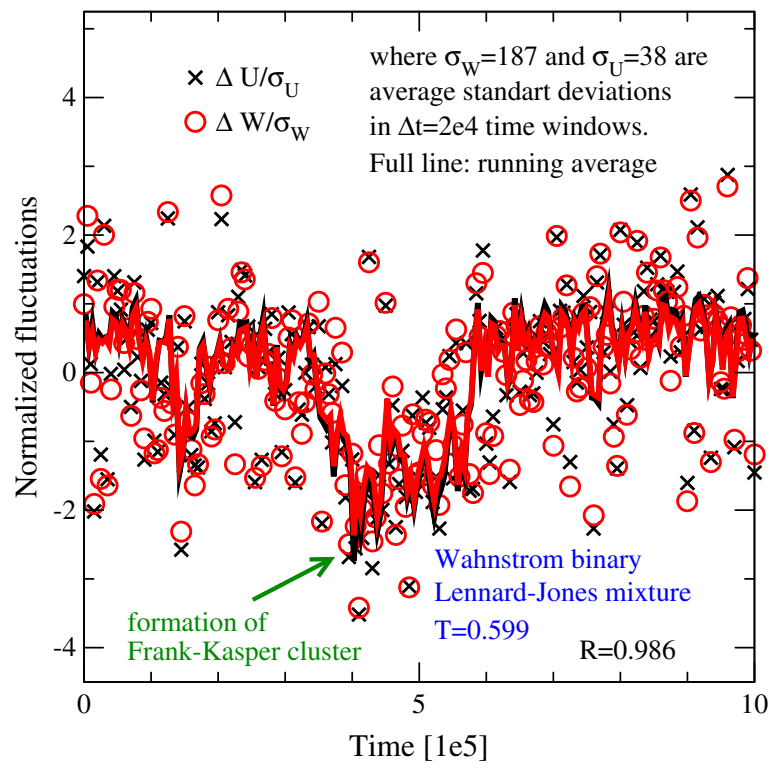

Fig. 1. (a) Instantaneous normalized equilibrium fluctuations of virial $W$ and potential energy $U$ in the standard single-component Lennard-Jones (LJ) liquid at constant volume (NVT simulation), showing that $W(t)$ and $U(t)$ correlate strongly. (b) The same for the supercooled Wahnström binary Lennard-Jones mixture [13]. For this liquid $W(t)$ and $U(t)$ correlate strongly even during the formation of a so-called Frank-Kaspers cluster [14]. (a)

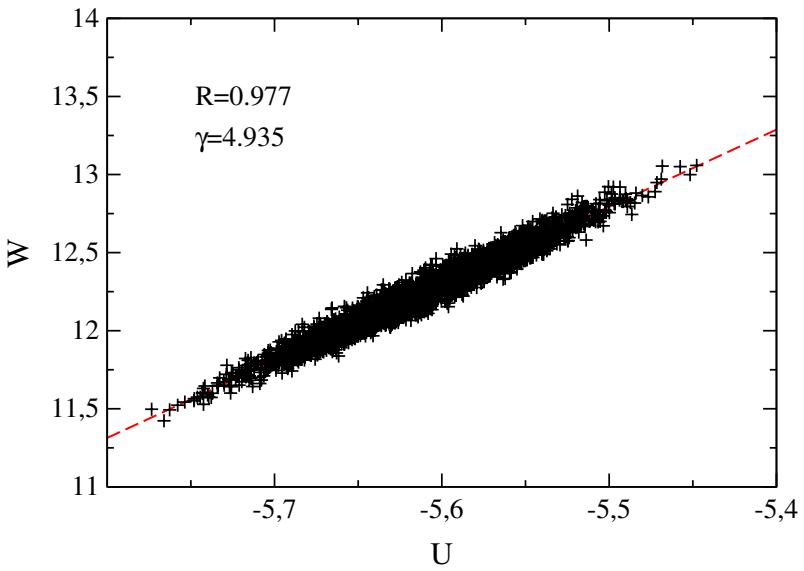

(b)

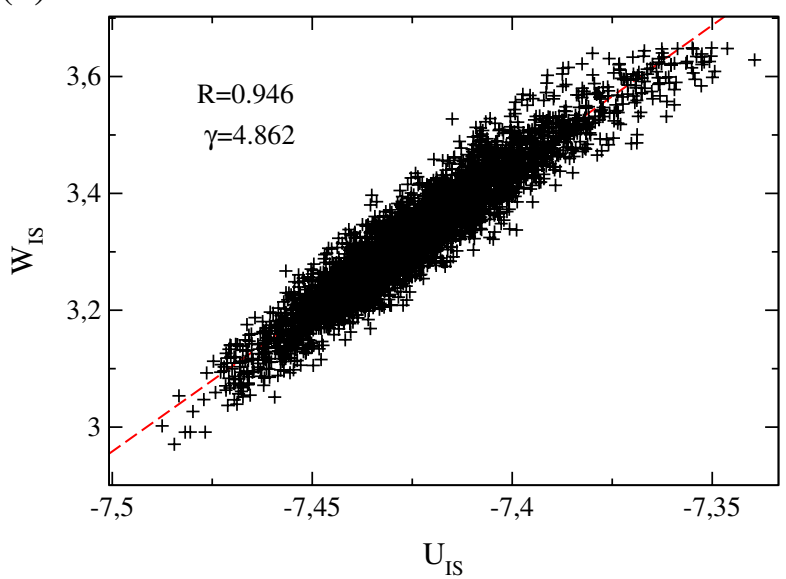

Fig. 2. (a) Scatter plot showing virial/potential energy correlations for the KobAndersen binary Lennard-Jones liquid (1000 particles studied by Monte Carlo simulation, $\rho=1.264, T=1.24$ in standard LJ units). (b) Inherent state energies and virials of the simulation in (a); the correlation is still high. The slope $\gamma$ defined by $\Delta W(t) \cong \gamma \Delta U(t)$ is slightly different, but close to that of the true dynamics ((a)).

The number $\gamma$, which varies slightly with state point, is roughly 6 for the standard $\mathrm{LJ}$ liquid, roughly 5 for the KABLJ liquid, and roughly 8 for the OTP model studied below in Fig. 7.

Since viscous liquid dynamics consists of long-time vibrations around potential energy minima - the so-called inherent states [18] followed by rapid transitions between the inherent states [19,20], it is interesting to study the inherent dynamics analogue of Fig. 2(a). This is done in Fig. 2(b), which gives the same simulation data after minimizing the configurations' potential energy using the conjugate gradient method. The correlations are still present and the "slope" $\gamma$ doesn't change very much - even though the virial decreased significantly going from (a) to (b). This illustrates the robustness of virial/potential energy correlations.

A convenient way to get an overview of a liquid's WU thermal equilibrium fluctuations at constant volume is to collect scatter plots for several state points in a common diagram. Fig. 3 (top) shows such a plot for the standard LJ liquid. Each state point is represented by a colored oval. As in Fig. 2 the strong correlation is reflected in the fact that the ovals are highly elongated. For each value of the density the ovals form almost straight lines with slope close to 6; in Ref. [4] it was shown that during and after constant-volume crystallization the system's scatter plots still fall on this line. The bottom three figures show the correlation coefficient $R$ (Eq. (3)), the "slope" $\gamma$, and the average pressure as functions of temperature for the different densities. Clearly, both $R$ and $\gamma$ are somewhat state-point dependent. 
(a)

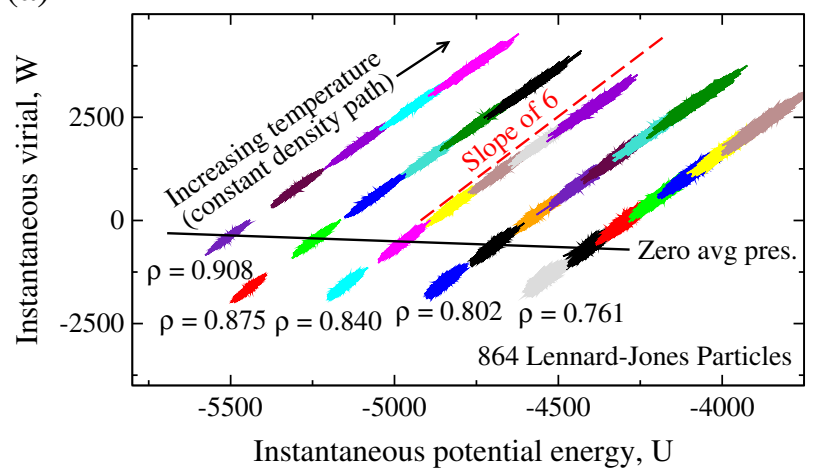

(b)

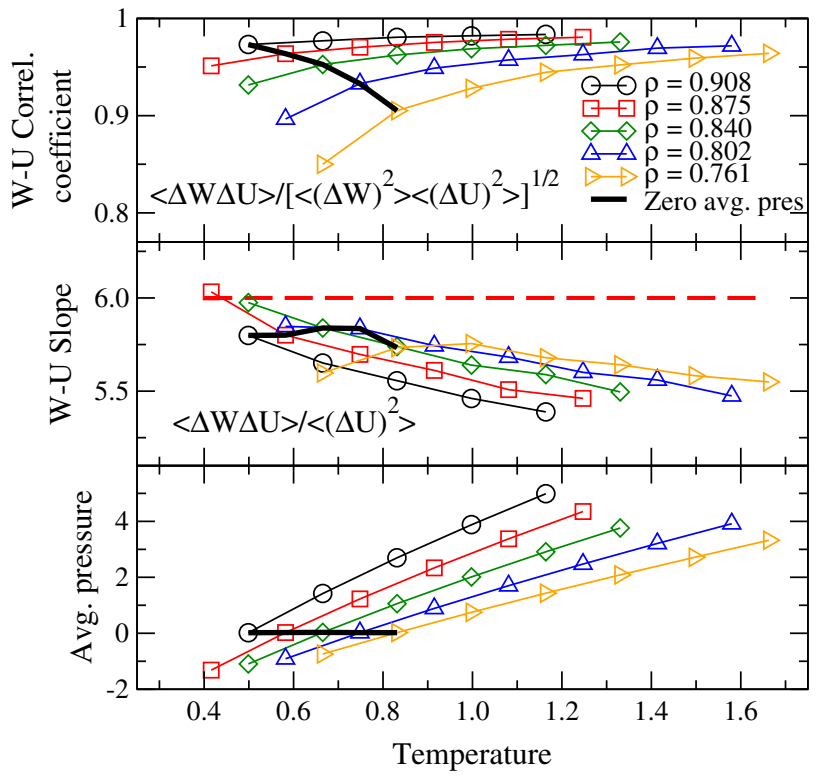

Fig. 3. (a) Scatter plot of the $W U$ thermal equilibrium fluctuations at constant volume for the standard single-component LJ liquid, and (b) plots of various quantities as functions of temperature for the different densities studied. The full black lines mark state points of zero average pressure.

At a given density $R$ increases with temperature whereas $\gamma$ decreases; at a given temperature $R$ increase with increasing density. The thick black lines mark state points of zero average pressure. Note that the density effect of increasing $R$ "wins" over the temperature effect of decreasing $R$ upon cooling at constant low-pressure. Thus one expects higher correlations upon supercooling a liquid. This is an important observation when the focus is on glass-forming liquids.

How common are strong WU correlations? In Ref. [3] we reported simulations of 13 different model liquids. All liquids with van der Waals type interactions were found to be strongly correlating $(R>0.9)$, whereas models of the two hydrogen-bonding liquids water and methanol were not. Although much remains to be done by means of theory and simulation, it has been established that liquids can be classified into two classes: (i) Strongly correlating liquids, a class that includes the van der Waals and metallic liquids; this liquid class appears to have a number of regularities and simple properties. (b) All remaining liquids - the hydrogen-bonded, the covalently bonded, and (strongly) ionic liquids; these liquids are generally much more complex.

\section{Cause of strong virial/potential energy correlations}

Before discussing the consequences of strong virial/potential energy correlations we reflect on the cause of the correlations.
The starting point is the well-known fact [7-11] that for any liquid in which the particles interact with purely repulsive inverse power law forces, $v(r) \propto r^{-n}$, there is $100 \%$ correlation between $W$ and $U: W(t)=\gamma U(t)$ where

$\gamma=\frac{n}{3}$

From the values of $\gamma$ close to 6 observed for the $\mathrm{LJ}$ liquid one would expect that, if the LJ liquid somehow corresponds to an IPL liquid, the exponent $n$ is close to 18 . At first sight this may seem strange given the $r^{-6}$ and $r^{-12}$ terms that enter into the definition of the $\mathrm{LJ}$ potential, but in fact a potential proportional to $r^{-18}$ does give a good fit to the repulsive part of the $\mathrm{LJ}$ potential (Fig. 4(a)). The reason that a much larger exponent than 12 is required is simply that the attractive $r^{-6}$ term makes the $\mathrm{LJ}$ repulsion much steeper than that of the $r^{-12}$ term alone. Fig. 4(b) shows that both potential energy and virial fluctuations of the $L J$ liquid are well represented by those of an $r^{-18}$ IPL potential.

In our first publications on strongly correlating liquids $[1,2]$ it was suggested that the strong correlations derive from particle close encounters taking the intermolecular distance to values below the $\mathrm{LJ}$

(a)

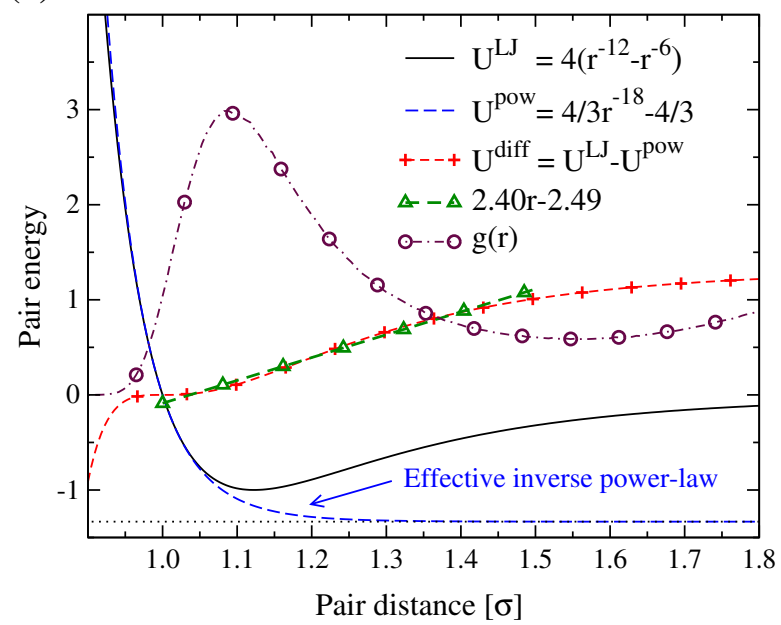

(b)

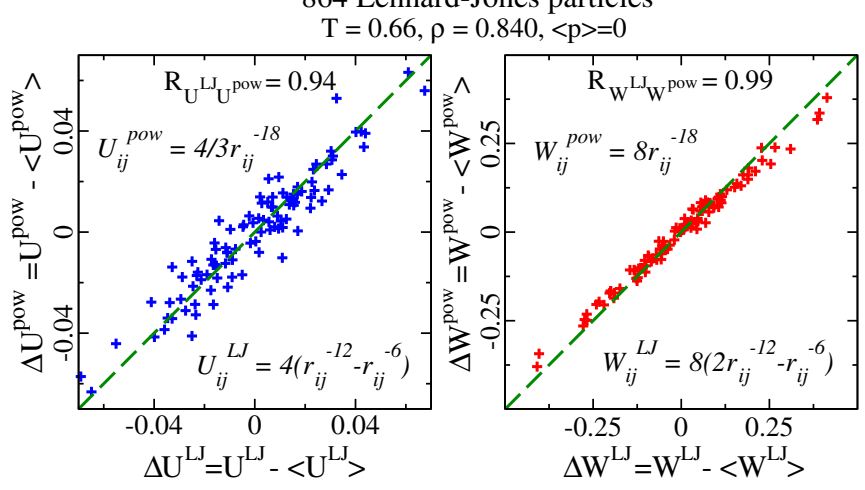

Fig. 4. (a) Approximation of the LJ potential by an effective inverse power law (IPL) potential $\propto r^{-18}$. The open circles mark the radial distribution function at a typical lowpressure state point. The blue dotted curve marks the IPL potential, which approximates the $\mathrm{LJ}$ potential quite well below the minimum. The difference between the LJ potential and the IPL potential is approximately linear in $r$, a fact which forms the basis for the "extended inverse power law" (eIPL) approximation (Eq. (7)) [4,5]. (b) Two figures demonstrating that the LJ potential energy and virial in their thermal equilibrium fluctuations both correlate strongly to the potential energy and virial for the $r^{-18}$ IPL potential. (For interpretation of the references to color in this figure legend, the reader is referred to the web version of this article.) 
potential minimum, at which the IPL potential is a good approximation. It quickly became clear, however, that this is not the full explanation; this cannot explain the existence of strong correlations in the crystal (above 99\% at low temperatures [4]) and the existence of correlations at low pressures, at which nearest-neighbor interparticle distances fluctuate around the LJ potential's minimum. Also, the original explanation is a single-pair explanation, which would imply that strong $W U$ correlations should be present as well in constant pressure ensembles. This contradicts our finding that switching from constant volume to constant pressure reduce $R$ from values above 0.9 to values around 0.1 [5].

References [4] and [5] detail the following more complete explanation of the cause of strong correlations. The difference between the IPL potential and the LJ potential is plotted in Fig. 4(a) as the red dashed curve. The green dashed curve is a straight line, which clearly approximates the red dashed curve well around the LJ minimum (i.e., over the entire first peak of the structure factor). Thus over the most important intermolecular distances an "extended" inverse power law potential, eIPL, defined by

$v_{\mathrm{eIPL}}(r)=A r^{-n}+B+C r$

gives a good approximation to the LJ potential. It has been shown by simulation that the linear term of the eIPL potential gives a contribution to the total potential energy that fluctuates little at constant volume $[4,5]$; likewise $W(t)$ fluctuations have little contribution from the linear term. Thus as regards fluctuations, the pure IPL gives representative results. This explains why the IPL approximation works well and why there are no longer any strong correlations when switching to constant pressure ensembles [4,5] (note that, in contrast to averages, fluctuations are generally ensemble dependent).

While the eIPL approximation explanation of strong WU correlations applies for most realistic cases, there are also strong correlations in the purely repulsive Weeks-Chandler-Andersen [21] version of the KABLJ liquid $[16,22,23]$. The slope $\gamma$ here varies significantly (from 5.0 to 7.5) over the range of densities and temperatures for which $\gamma$ is fairly constant for the KABLJ liquid. Our simulations show, interestingly, that the strong WU correlations for the WCA case is a singleparticle effect, not a cooperative effect that only applies at constant volume conditions as observed for LJ-type liquids. More work is needed to illuminate the correlation properties of this interesting potential.

\section{Isomorphs: Curves of invariance in the phase diagram}

This section defines isomorphs and summarizes their invariants. An isomorph is a curve in the phase diagram along which a large number of properties are invariant. As shown in Ref. [6] a liquid has isomorphs if and only if the liquid is strongly correlating.

For any microscopic configuration $\left(\mathbf{r}_{1}, \ldots, \mathbf{r}_{N}\right)$ of a thermodynamic state point with density $\rho$, the "reduced" coordinates are defined by $\tilde{\mathbf{r}}_{i}=\rho^{1 / 3} \mathbf{r}_{i}$. Two state points (1) and (2) with temperatures $T_{1}$ and $T_{2}$ and densities $\rho_{1}$ and $\rho_{2}$ are said to be isomorphic [6] if, whenever two microscopic configurations $\left(\mathbf{r}_{1}^{(1)}, \ldots, \mathbf{r}_{N}^{(1)}\right)$ and $\left(\mathbf{r}_{1}^{(2)}, \ldots, \mathbf{r}_{N}^{(2)}\right)$ have identical reduced coordinates, to a good approximation they have proportional configurational NVT Boltzmann probability factors:

$e^{-U\left(\mathbf{r}_{1}^{(1)}, \ldots, \mathbf{r}_{N}^{(1)}\right) / k_{B} T_{1}}=C_{12} e^{-U\left(\mathbf{r}_{1}^{(2)}, \ldots, \mathbf{r}_{N}^{(2)}\right) / k_{B} T_{2}}$

The constant $C_{12}$ depends only on the state points (1) and (2), not on the microscopic configurations. Isomorphic curves in the state diagram are defined as curves for which any two state points are isomorphic. The property of being isomorphic defines a mathematical equivalence relation on the set of state points and "isomorphs" are the corresponding equivalence classes.
The property of having isomorphs is generally approximate - only IPL liquids have exact isomorphs. For this reason Eq. (8) should be read as obeyed, not rigorously, but to a good approximation, and merely for the "physically relevant" configurations, i.e., those that do not have negligible canonical probabilities [6].

Fig. 5 illustrates Eq. (8) by checking the logarithm of this equation, where (a) gives simulation data for the KABLJ liquid. We consider several microscopic configurations of the state point with density and temperature $\left(\rho_{1}, T_{1}\right)=(1.258,0.628)$ in standard LJ units. For these configurations the total potential energy was evaluated. In order to investigate whether the state point has an isomorphic state point at density $\rho_{2}=1.228$, we scaled the simulated configurations of the initial state point to density $\rho_{2}$. For the scaled configurations the potential energies are plotted against the original potential energies of state point 1 (left panel of Fig. 5(a)). According to the isomorph definition Eq. (8) the best fit slope gives the ratio between the temperatures of the isomorphic state points; in this way we find that $T_{2}=0.555$.

The right panel of Fig. 5(a) investigates the consistency of this procedure by reversing it in order to check whether the original temperature $T_{1}$ is arrived at. When this is done, one does find the original temperature (0.628). Two things should be noted. The first is the very strong correlation between the potential energies of the scaled configurations, as required for having good isomorphs. The second noticeable fact is that the best fit lines do not pass through $(0,0)$, showing that the constant $C_{12}$ of Eq. ( 8 ) is not unity as it would be for an IPL liquid ( $C_{12}$ is determined by the contribution to the partition function coming from the linear term of the eIPL (Eq. (7), and thus $C_{12}$ reflects the deviation from true IPL behavior).

Fig. 5(b) shows results for the same "direct isomorph test" for the non-strongly correlating liquid SPC water, starting from temperature $T_{1}=200 \mathrm{~K}$. From the slope of the left panel we find $T_{2}=179.6 \mathrm{~K}$. When the reverse jump is performed, however, one does not come back to the initial state point, but to a predicted temperature of $166.4 \mathrm{~K}$. This shows that water does not have isomorphs, which is consistent with the fact that water is not a strongly correlating liquid [3].

For the practical identification of an isomorph in the phase diagram the above method may be used. Alternatively, it has been shown [6] that to a good approximation isomorphs are characterized by

$\frac{\rho^{\gamma}}{T}=$ Const.

Here $\gamma$ is the above discussed "slope" characterized by $\Delta W(t) \cong \gamma \Delta U(t)$. As shown in Ref. [6] this quantity may be calculated to a good approximation from the equilibrium fluctuations at one single state point via the following expression that, incidentally, also gives the least-squared linearregression best fit slope of $W U$ scatter plots (compare Appendix B of Ref. [3]):

$\gamma=\frac{<\Delta W \Delta U>}{<(\Delta U)^{2}>}$.

Several physical quantities are invariant to a good approximation along a strongly correlating liquid's isomorphs [6]. These include: 1) Thermodynamic properties like the excess entropy (i.e., in excess of the ideal gas entropy at same density and temperature) and the excess isochoric specific heat, 2) static averages like the radial distribution function(s) in reduced coordinates, 3) dynamic quantities in properly reduced units like the diffusion constant, viscosity, and heat conductivity, 4) time-autocorrelation functions, 5) average reduced relaxation times, etc.

Fig. 6 shows results of simulations of the KABLJ liquid at two isomorphic and two isothermal state points of the AA particle radial distribution functions and the AA incoherent intermediate scattering 
(a)
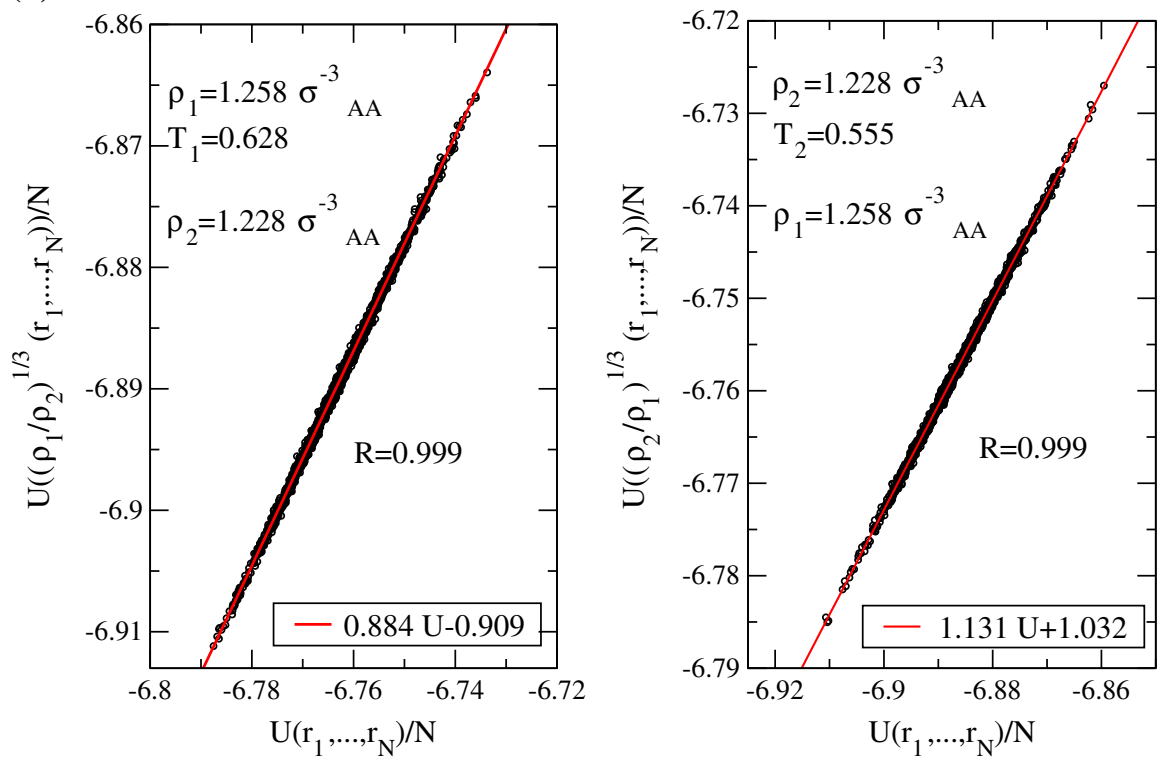

(b)
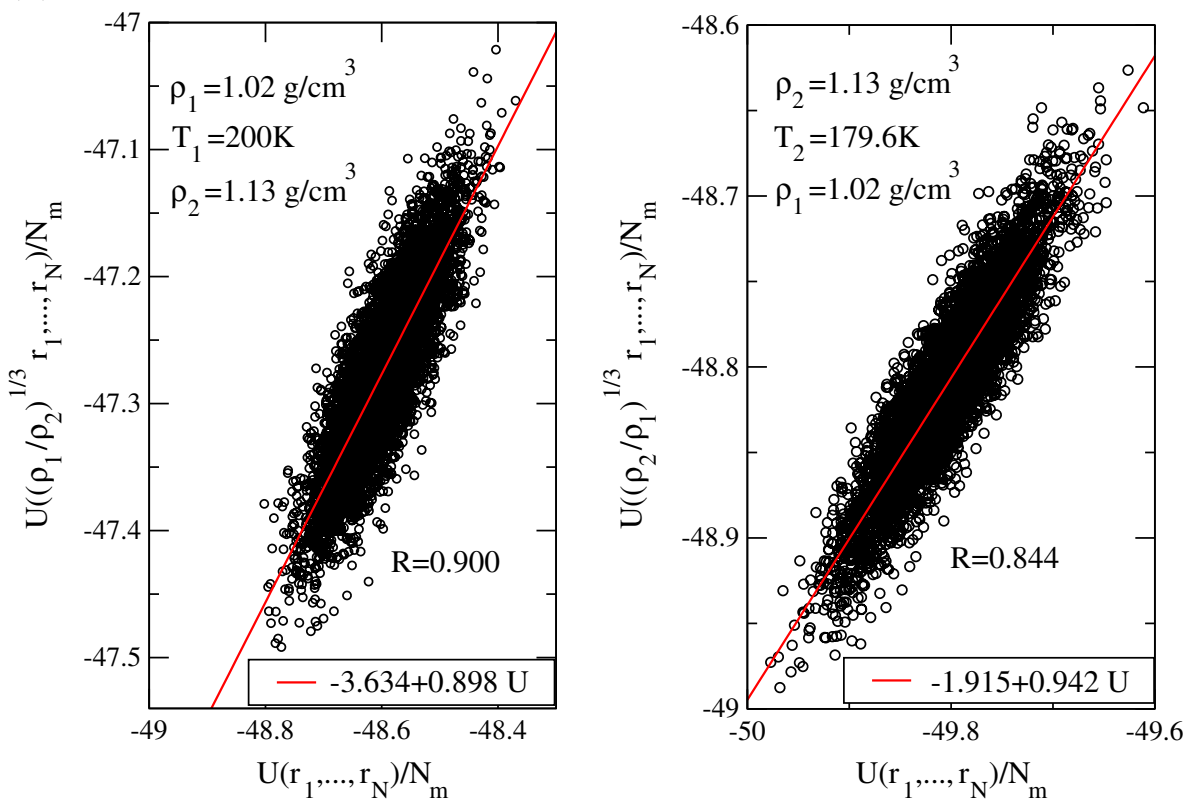

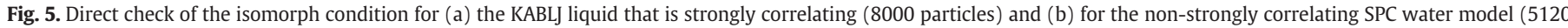

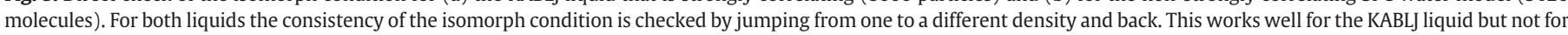
SPC water; details are given in the text.

functions, respectively. These figures confirm the prediction that isomorphic state points have almost identical static distribution functions and almost identical dynamics.

Since the isochoric specific heat is an isomorph invariant, this quantity should be a function of $\rho^{\gamma} / T$ for a strongly correlating liquid. Fig. 7 confirms this prediction for the Lewis-Wahnström OTP model consisting of three LJ spheres [24].

The theory of isomorphs [6] further predicts that jumps between two isomorphic state points should take the system instantaneously to equilibrium, because the Boltzmann statistical factors of two isomorphic state points are proportional. More generally, isomorphic state points are equivalent during any aging scheme. We recently showed [26] that the isomorph concept throws light on the concept of an effective temperature, which can be calculated from the violation of the fluctuation-dissipation theorem. In particular, the isomorph theory implies that for strongly correlating liquids the effective temperature - after a jump to a new (low) temperature and a new density - depends only on the new density (Fig. 8(a)). This does not apply for the non-strongly correlating monatomic Lennard-Jones Gaussian liquid, confirming the general conjecture that strongly correlating liquids have simpler physics than liquids in general [26]. Fig. 8(b) shows a result confirming the finding of Ref. [26] that the effective temperature concept for a strongly correlating liquid makes good sense physically. On the $\mathrm{x}$-axis the inherent state energies of given state points are shown as the system fell out of equilibrium. The arrested phase is characterized by an effective temperature $T_{\text {eff }}[26]$. On the y-axis is shown the inherent energies found from an equilibrium simulation with temperature equal to $T_{\text {eff }}$ for the corresponding arrested phase. The red points give data for the strongly correlating KABLJ liquid, the 
(a)

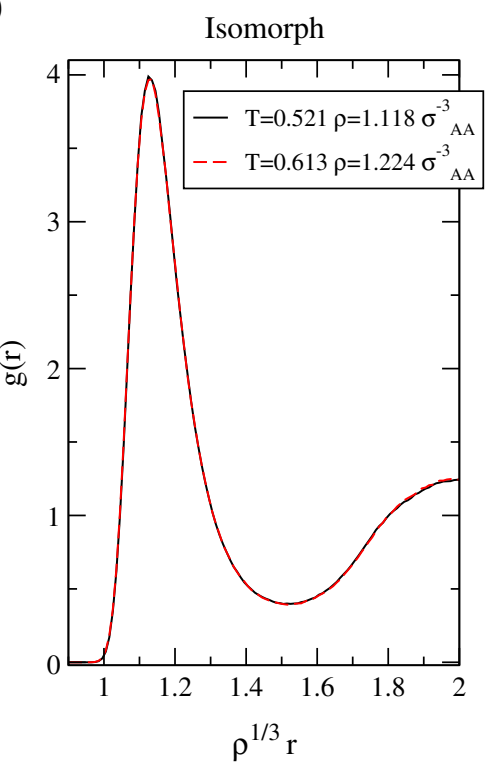

(b)

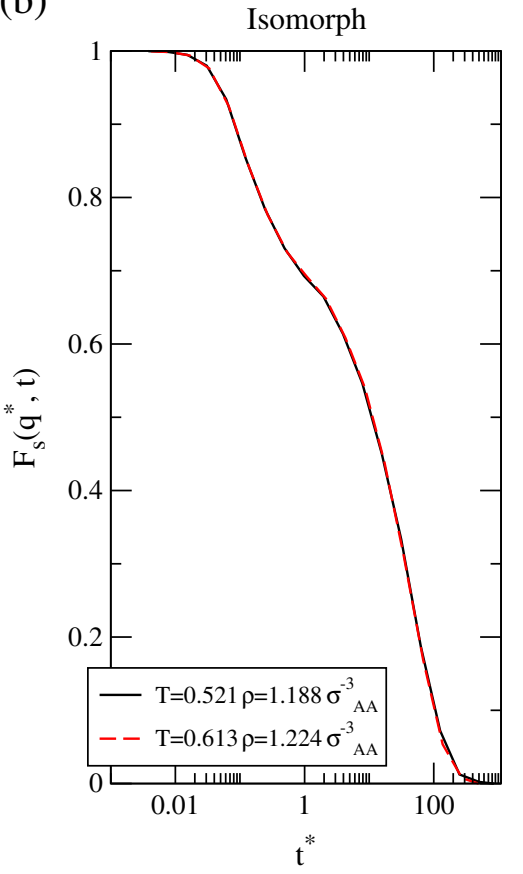

Isotherm
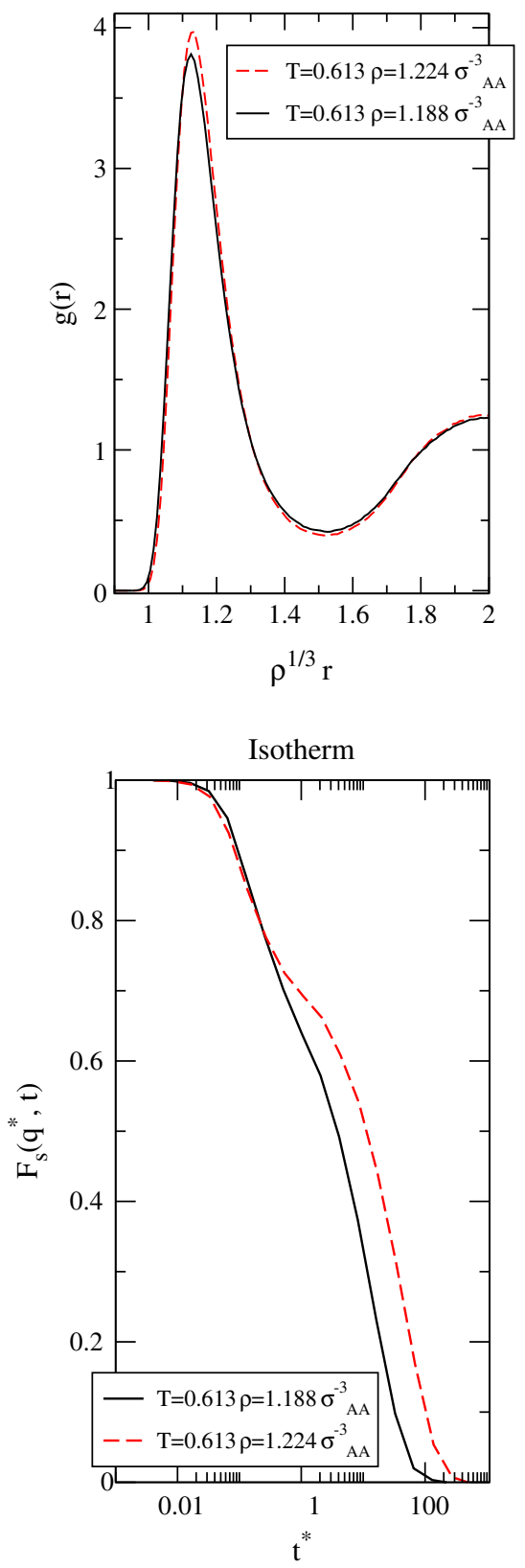

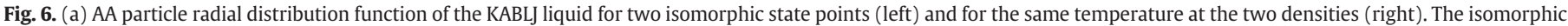

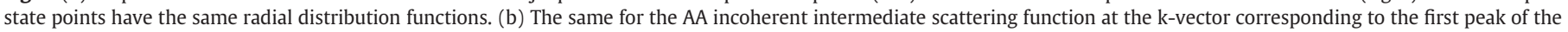
radial distribution function. The two isomorphic state points have the same dynamics in reduced units (used here).

green points give data for the non-strongly correlating monatomic Lennard-Jones Gaussian liquid. Clearly, the latter system fell out of equilibrium by freezing into a part of phase space that is not

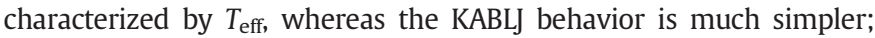
here it appears that basically the same regions of phase space are explored during aging as in equilibrium. More work is needed, however, to detail this point, which at present is merely a conjecture consistent with above discussed findings.

\section{Are strongly correlating liquids merely approximate IPL liquids?}

The only liquids having 100\% exact isomorphs are IPL liquids, i.e., liquids where the potential energy is an Euler homogeneous function of the particle coordinates. Note that this case includes liquids that not just have pair interactions; interactions may well be of three- or fourbody type as long as all terms in $U$ scale with density in the same way. The fact that only IPL liquids have exact isomorphs and the fact that the LJ potential's repulsion is well approximated by an IPL term $\left(r^{-18}\right)$ has led to speculations that all strongly correlating liquids are "just" approximate IPL liquids. In our opinion, for the below reasons, this is not a useful way to thinking of this large liquid class.

First of all, it should be noted that the density scaling exponent $\gamma$ usually varies throughout the phase diagram, as well as along isomorphs. It may be shown that for any liquid with good isomorphs $\gamma$ varies only with volume [6], but since volume changes along an isomorph, $\gamma$ may do this as well. A characteristic of the isomorph definition is that it does not refer to approximate exponents (or to 


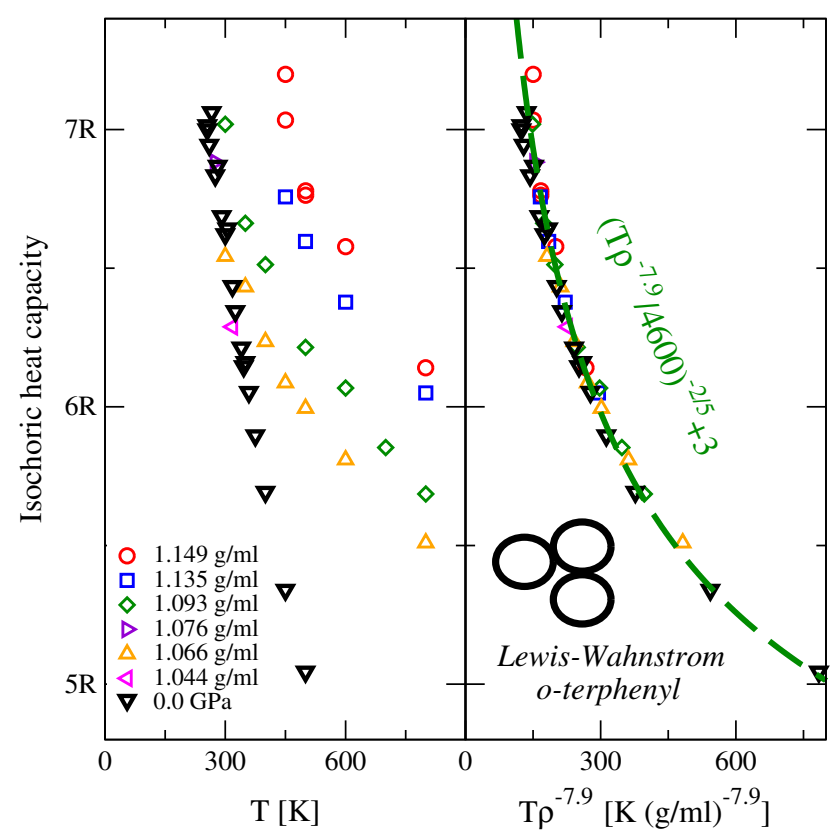

Fig. 7. $c_{V}$ per particle for the Lewis-Wahnström model of ortho-terphenyl consisting of three LJ spheres arranged with fixed bond lengths [24]. The left panel shows the simulation data, the right panel shows the same data replotted as function of $\rho^{7.9} / T$ in which the exponent 7.9 was determined from the proportionality between equilibrium virial and potential energy fluctuations [12]. This model follows the RosenfeldTarazona prediction of $c_{V}$ varying with temperature as $T^{-2 / 5}$ asymptotically at low temperatures [25].

correlations). For the $\mathrm{LJ}$ liquid, while $\gamma$ at typical state points is close to 6 , at high temperatures/pressures it approaches the value 4 coming from the repulsive $r^{-12}$ term.

Secondly, it is important to emphasize that while all general isomorph invariants are also invariants for IPL liquids along the curves of constant $\rho^{n / 3} / T$, several such IPL invariants are not general isomorph invariants. Examples are $[5,6]$ (where all below quantities are excess quantities):

1. The free energy over temperature, $F / T$.

2. The energy over temperature, $U / T$.

3. The pressure times volume over temperature, $p V / T$.

4. The constant-temperature compressibility times density times temperature, $\kappa_{T} \rho T$.

The reason that these (and some other) IPL invariants do not generalize to become isomorph invariants is that the constant $C_{12}$ of Eq. (8) is not unity in general, whereas for all IPL liquids one trivially has $C_{12}=1$. The constant $C_{12}$ is expected to generally vary with density, because it derives from the contribution to the free energy coming from the linear term in the eIPL potential (Eq. (7)). Some IPL invariants like the (excess) entropy or the (excess) $C_{V}$ only depend on the canonical probabilities, for which the value of $C_{12}$ is irrelevant because the Boltzmann factors are normalized; these IPL invariants generalize to become isomorph invariants. Other IPL invariants - those involving only volume derivatives of the free energy - in general get contributions from $C_{12}$ for strongly correlating liquids; they do not give rise to general isomorph invariants.

\section{Some experimental predictions for strongly correlating glass-forming liquids}

Strongly correlating liquids have a number of properties since long discussed in the literature in various contexts. For instance, since the melting line in the phase diagram is an isomorph [6], strongly correlating liquids have several invariants along their melting lines, including the radial distribution function(s) and dimensionless transport coefficients. (a)

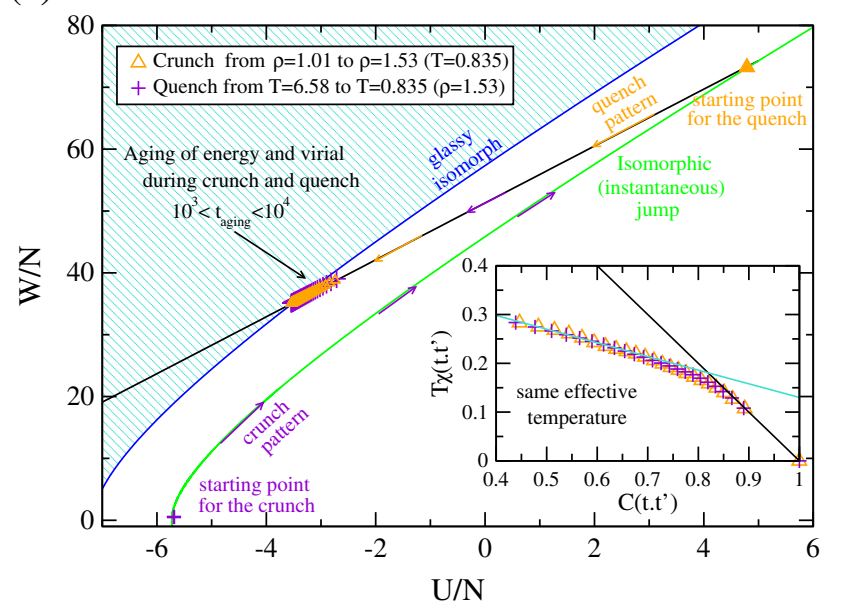

(b)

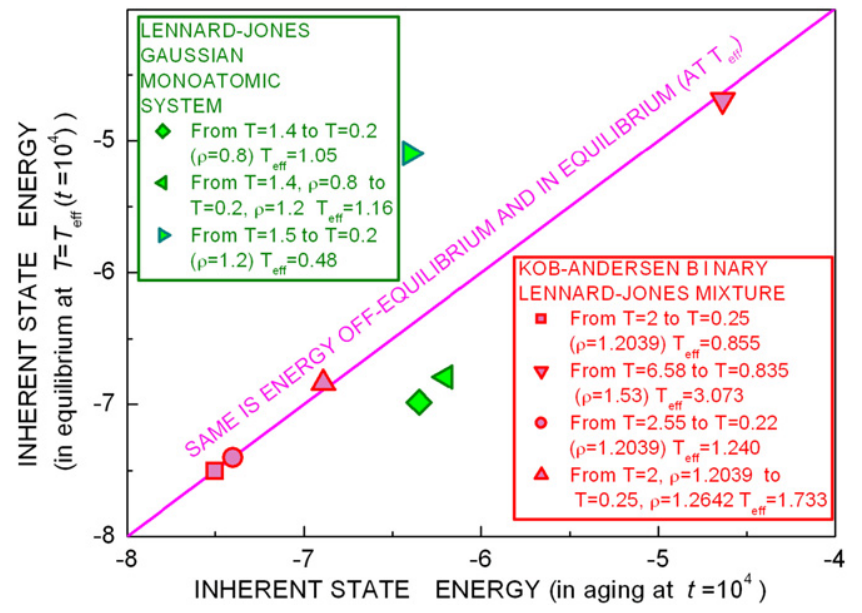

Fig. 8. (a) Virial versus potential energy for the KABLJ liquid during a temperature quench and a crunch [26]. A crunch increases the density and keeps the temperature constant; as shown in Ref. [10] this is equivalent to an instantaneous jump along an isomorph (green curve) to the right density followed by a temperature quench (black curve). The inset shows that the crunch and the quench have the same fluctuationdissipation violation factors if they end at same density (i.e., the two jumps result in the same effective temperature). (b) Inherent state energies for several state points of the KABLJ liquid as the system falls out of equilibrium and freezes versus the equilibrium inherent state energy at the effective temperature (details are given in Ref. [10], from which online supporting material (b) is reproduced). (For interpretation of the references to color in this figure legend, the reader is referred to the web version of this article.)

Such regularities have been observed in simulation and experiment, and the known exceptions appear always to involve liquids that are not strongly correlating; we refer to the reader to Ref. [4] for more details. This section focuses on predictions for highly viscous liquids, for which the strong correlation property implies the following experimental predictions:

\subsection{Density scaling}

In the last decade, in particular since 2005, many papers appeared dealing with density scaling, i.e., the finding that for several glassforming liquids the relaxation time $\tau$ at varying pressure and temperature is a function of the quantity $\rho^{\gamma} / T$ where exponent $\gamma$ is an empirical fitting parameter,

$\tau=F\left(\rho^{\gamma} / T\right)$. 
Neither the function $F$ nor the exponent $\gamma$ are universal. The isomorph theory $[6,12]$ shows that all strongly correlating glass formers obey density scaling with the exponent $\gamma$ given by the equilibrium fluctuations at one state point (Eq. (10)) provided $\gamma$ is fairly constant over the relevant part of phase space. This is consistent with the experimental finding that density scaling works well for van der Waals liquids [27,28], but not for hydrogen-bonded liquids [29-32]. Density scaling has been shown to apply in computer simulations of strongly correlating liquids with $\gamma$ given by Eq. (10) to a good approximation $[12,33]$.

\subsection{Isochronal superposition}

Isochronal superposition is the further recent finding that for varying pressures and temperatures the dielectric loss as a function of frequency depends only on the loss-peak frequency [34,35]. This is trivial, of course, if the liquid obeys time-temperature-pressure superposition (TTPS) in which case nothing changes. But many liquids do not obey TTPS, and for such liquids isochronal superposition is a new and striking regularity, which is found to work generally for van der Waals liquids, but not for hydrogen-bonding liquids [35]. Since both the relaxation time and the relaxation spectrum are isomorph invariants [6], isochronal superposition must apply for any strongly correlating liquid: If temperature and pressure for two state points are such that their relaxation times are the same, the two points must belong to the same isomorph. Thus they must have same relaxation time spectra for any observable, for instance the normalized dielectric loss as function of (reduced) frequency should be the same.

\subsection{Frequency-dependent viscoelastic response functions}

There are eight fundamental complex, frequency-dependent linear thermoviscoelastic response functions like, e.g., the frequency-dependent isochoric or isobaric specific heat, the frequencydependent isobaric expansion coefficient, and the frequency-dependent adiabatic or isothermal compressibility [36]. Standard linear irreversible thermodynamics arguments, where the Onsager relations play the role of the Maxwell relations of usual thermodynamics, show that there are only three independent frequency-dependent response functions. If stochastic dynamics is assumed, as is realistic for highly viscous liquids [37], there are only two independent response functions [38-40]. For strongly correlating liquids a further simplification applies, however, namely that there is just a single independent response function $[1,2,37,42]$. Since there are explicit expressions linking the different response functions (depending on the ensemble considered [36]), this can be tested experimentally. Unfortunately it is difficult to measure thermoviscoelastic functions properly; to the best of our knowledge there are yet no reliable data for a complete set (three or more) of such response functions for any liquid.

\subsection{The Prigogine-Defay ratio: Strongly correlating liquids as approximate} single-parameter liquids

After many years of little interest the Prigogine-Defay (PD) ratio [42-44] has recently again come into focus in the scientific discussion about glass-forming liquids [36,45-48]. From a theoretical perspective the PD ratio is poorly defined since it involves extrapolations from the liquid and glass phases to a common temperature [36,41]. It is possible to overcome this problem by modifying the PD ratio by referring exclusively to linear response experiments [36]; here the traditional difference between liquid and glass responses is replaced by a difference between low- and high-frequency values of the relevant frequency-dependent thermoviscoelastic response function. In this formulation, the property of strong virial/potential energy correlations manifests itself as a PD ratio close to unity. Indeed, an extensive compilation of data showed that van der Waals bonded liquids and polymers have PD ratios close to unity [49].

The theoretical developments of Refs. $[2,4,36]$ show that in any reasonable sense of the old concept "single-order-parameter liquid", strongly correlating liquids are precisely the single-order-parameter liquids. The isomorph concept makes this even clearer: State points along an isomorph have so many properties in common that they are identical from many viewpoints. In the two-dimensional phase diagram this leaves just one parameter to classify which isomorph the state point is on. Thus a liquid with (good) isomorphs is an (approximate) single-parameter liquid. Note that this is consistent with the old viewpoint that single-parameter liquids should have unity PD ratio [42-44].

\subsection{Cause of the relaxation time's non-Arrhenius temperature dependence: The isomorph filter}

Since the relaxation time $\tau$ is an isomorph invariant for any strongly correlating liquid, any universally valid theory predicting $\tau$ to depend on some physical quantity must give $\tau$ as a function of another isomorph invariant (we do not distinguish between the relaxation time and the reduced relaxation time since their temperature dependencies are virtually identical). This gives rise to an "isomorph filter" [6], showing that several well-known models cannot be universally valid. For instance, the entropy model cannot apply in the form usually used by experimentalists: $\tau \propto \exp \left(C / S_{\text {conf }} T\right)$ where $C$ is a constant and $S_{\text {conf }}$ is the configurational entropy; it can only be correct if $C$ varies with density as $C \propto \rho^{\gamma}$. Likewise, the free volume model does not survive the isomorph filter unless the threshold free volume scales with inverse density. As an example of the isomorph filter, note that if the characteristic volume $V_{c}$ of the shoving model (which predicts that $\tau \propto \exp \left(V_{c} G_{\infty} / k_{B} T\right)$ [50-52])) varies with density as $V_{c} \propto 1 / \rho$, this model is consistent with the isomorph filter. However, replacing $G_{\infty}$ by the instantaneous bulk modulus $K_{\infty}$ would lead to an expression that is not isomorph invariant.

\subsection{Fictive temperature variations following a temperature jump}

Any jump from equilibrium at some density and temperature to another density and temperature proceeds as if the system first jumped along an isomorph to equilibrium at the final density and then, starting immediately thereafter, jumped to the final temperature (Fig. 8(a)): The first isomorphic jump takes the system instantaneously to equilibrium. This applies for all strongly correlating liquids and means that glass-forming van der Waals and metallic liquids are predicted to have simpler aging behavior than, e.g., covalently bonded liquids like ordinary oxide glasses [26].

In traditional glass science the concept of "fictive temperature" is used as a structural characteristic that by definition gives the temperature at which the structure would be in equilibrium [53-58]. In glass science, for any aging experiment one assumes that the fictive temperature adjusts itself monotonically from the initial temperature to the final temperature. Consider, however, a sudden temperature increase applied at ambient pressure. In this case there is first a rapid thermal expansion before any relaxation takes place. This "instantaneous isomorph" takes the system initially to a state with canonical (Boltzmann) probability factors corresponding to a lower temperature. In other words, immediately after a temperature up jump the system has a structure which is characteristic of a temperature that is lower than the initial temperature. Thus with any reasonable definition of the fictive temperature, this quantity must initially decrease during an isobaric temperature jump up - at least for all strongly correlating liquids. 


\section{Summary}

The class of strongly correlating liquids includes the van der Waals and metallic liquids, but excludes the hydrogen-bonded, the covalently bonded, and the (strongly) ionic liquids. Due to their "hidden scale invariance" - the fact that strongly correlating liquids inherit a number of IPL properties - these liquids are simpler than liquids in general. Strongly correlating liquids are characterized by having isomorphs to a good approximation, curves along which a number of physical properties are invariant when given in properly reduced units. In particular, for glass-forming liquids the property of strong virial/potential energy correlations in the equilibrium fluctuations implies a number of experimental predictions. Some of these, like density scaling and isochronal superposition, are well-established experimental facts for van der Waals liquid and known not apply for hydrogen-bonded liquids. This is predicted by the theory of isomorphs. Some of the predicted properties have not yet been tested experimentally, for instance that the density scaling exponent can be determined by measuring the linear thermoviscoelastic response functions at a single state point, or that jumps between isomorphic state points take the system instantaneously to equilibrium, no matter how long is the relaxation time of the liquid at the relevant state points. - Hopefully, this paper may inspire to experiments testing the new predictions.

\section{Acknowledgments}

URP is supported by the Danish Council for Independent Research in Natural Sciences. The Center for Viscous Liquid Dynamics "Glass and Time" is sponsored by the Danish National Research Foundation (DNRF).

\section{References}

[1] U.R. Pedersen, N.P. Bailey, T.B. Schrøder,J.C. Dyre, Phys. Rev. Lett. 100 (2008) 015701

[2] U.R. Pedersen, T. Christensen, T.B. Schrøder, J.C. Dyre, Phys. Rev. E 77 (2008) 011201.

[3] N.P. Bailey, U.R. Pedersen, N. Gnan, T.B. Schrøder, J.C. Dyre, J. Chem. Phys. 129 (2008) 184507

[4] N.P. Bailey, U.R. Pedersen, N. Gnan, T.B. Schrøder, J.C. Dyre, J. Chem. Phys. 129 (2008) 184508

[5] T.B. Schrøder, N.P. Bailey, U.R. Pedersen, N. Gnan, J.C. Dyre, J. Chem. Phys. 131 (2009) 234503

[6] N. Gnan, T.B. Schrøder, U.R. Pedersen, N.P. Bailey, J.C. Dyre, J. Chem. Phys. 131 (2009) 234504

[7] D. Chandler, Introduction to Modern Statistical Mechanics, Oxford University Press, 1987.

[8] L.D. Landau, E.M. Lifshitz, Statistical Physics, Part I, Pergamon Press, London, 1980.

[9] M.P. Allen, D.J. Tildesley, Computer Simulation of Liquids, Oxford Science Publications, Oxford, 1987

[10] L.E. Reichl, A Modern Course in Statistical Physics, 2nd ed. Wiley, New York, 1998.
[11] J.P. Hansen, J.R. McDonald, Theory of Simple Liquids, 3ed Academic, New York, 2005.

[12] T.B. Schrøder, U.R. Pedersen, N.P. Bailey, S. Toxvaerd, J.C. Dyre, Phys. Rev. E 80 (2009) 041502.

[13] G. Wahnström, Phys. Rev. A 44 (1991) 3752.

[14] U.R. Pedersen, T.B. Schrøder, J.C. Dyre, P. Harrowell, Phys. Rev. Lett. 104 (2010) 105701.

[15] U.R. Pedersen, G.H. Peters, T.B. Schrøder, J.C. Dyre, J. Phys. Chem. B 114 (2010) 2124.

[16] W. Kob, H.C. Andersen, Phys. Rev. Lett. 73 (1994) 1376.

[17] S. Toxvaerd, U.R. Pedersen, T.B. Schrøder, J.C. Dyre, J. Chem. Phys. 130 (2009) 224501.

[18] F.H. Stillinger, T.A. Weber, Phys. Rev. A 28 (1983) 2408.

[19] M. Goldstein, J. Chem. Phys. 51 (1969) 3728.

[20] T.B. Schrøder, S. Sastry, J.C. Dyre, S.C. Glotzer, J. Chem. Phys. 112 (2000) 9834

[21] J.D. Weeks, D. Chandler, H.C. Andersen, J. Chem. Phys. 54 (1971) 5237.

[22] L. Berthier, G. Tarjus, Phys. Rev. Lett. 103 (2009) 170601.

[23] D. Coslovich, C.M. Roland, J. Chem. Phys. 131 (2009) 151103.

[24] L.J. Lewis, G. Wahnström, Phys. Rev. E 50 (1994) 3865.

[25] Y. Rosenfeld, P. Tarazona, Mol. Phys. 95 (1998) 141.

[26] N. Gnan, C. Maggi, T.B. Schrøder, J.C. Dyre, Phys. Rev. Lett. 104 (2010) 125902.

[27] C. Alba-Simionesco, A. Cailliaux, A. Alegria, G. Tarjus, Europhys. Lett. 68 (2004) 58.

[28] C.M. Roland, S. Hensel-Bielowka, M. Paluch, R. Casalini, Rep. Prog. Phys. 68 (2005) 1405.

[29] A. Grzybowski, K. Grzybowska, J. Ziolo, M. Paluch, Phys. Rev. E 74 (2006) 041503.

[30] C.M. Roland, S. Bair, R. Casalini, J. Chem. Phys. 125 (2006) 124508.

[31] A. Le Grand, C. Dreyfus, C. Bousquet, R.M. Pick, Phys. Rev. E 75 (2007) 061203.

[32] C.M. Roland, R. Casalini, R. Bergman, J. Mattsson, Phys. Rev. B 77 (2008) 012201

[33] D. Coslovich, C.M. Roland, J. Chem. Phys. 130 (2009) 014508.

[34] C.M. Roland, R. Casalini, M. Paluch, Chem. Phys. Lett. 367 (2003) 259.

[35] K.L. Ngai, R. Casalini, S. Capaccioli, M. Paluch, C.M. Roland, J. Phys. Chem. B 109 (2005) 17356.

[36] N.L. Ellegaard, T. Christensen, P.V. Christiansen, N.B. Olsen, U.R. Pedersen, T.B Schrøder, J.C. Dyre, J. Chem. Phys. 126 (2007) 074502.

[37] T. Gleim, W. Kob, K. Binder, Phys. Rev. Lett. 81 (1998) 4404.

[38] J. Meixner and H. G. Reik, Principen der Thermodynamik und Statistik (Handbuch der Physik) vol 3, ed. S. Flügge (Springer, Berlin, 1959) p. 413.

[39] P.K. Gupta, C.T. Moynihan, J. Chem. Phys. 65 (1976) 4136.

[40] J.I. Berg, A.R. Cooper, J. Chem. Phys. 68 (1978) 4481.

[41] N.P. Bailey, T. Christensen, B. Jakobsen, K. Niss, N.B. Olsen, U.R. Pedersen, T.B. Schrøder, J.C. Dyre, J. Phys. Condens. Matter 20 (2008) 244113.

[42] I. Prigogine, R. Defay, Chemical Thermodynamics, Longman, London, 1954.

[43] R.O. Davies, G.O. Jones, Proc. Roy. Soc. 217 (1952) 26.

[44] R.O. Davies, G.O. Jones, Adv. Phys. 2 (1953) 370.

[45] Th.M. Nieuwenhuizen, Phys. Rev. Lett. 79 (1997) 1317

[46] J.W.P. Schmelzer, I. Gutzow, J. Chem. Phys. 125 (2006) 184511.

[47] L. Wondraczek, H. Behrens, J. Chem. Phys. 127 (2007) 154503.

[48] R.M. Pick, J. Chem. Phys. 129 (2008) 124115.

[49] U. R. Pedersen, Ph. D. thesis, Roskilde University (2009) [available at http://www urp.dk/phdthesis.htm].

[50] J.C. Dyre, N.B. Olsen, T. Christensen, Phys. Rev. B 53 (1996) 2171.

[51] J.C. Dyre, Rev. Mod. Phys. 78 (2006) 953.

[52] D.H. Torchinsky, J.A. Johnson, K.A. Nelson, J. Chem. Phys. 130 (2009) 064502.

[53] A.J. Kovacs, Fortschr. Hochpoly.-Forsch. 3 (1963) 394.

[54] C.T. Moynihan, et al., Ann. NY Acad. Sci. 279 (1976) 15.

[55] O.V. Mazurin, J. Non-Cryst. Solids 25 (1977) 129.

[56] L.C.E. Struik, Physical Aging in Amorphous Polymers and Other Materials, Elsevier, Amsterdam, 1978.

[57] G.B. McKenna, J. Res. Natl. Inst. Stand. Technol. 99 (1994) 169.

[58] I.M. Hodge, Science 267 (1995) 1945. 